\title{
The Feshbach-Schur map and perturbation theory
}

\author{
Geneviève Dusson Israel Michael Sigal Benjamin Stamm
}

To Ari with friendship and admiration

\begin{abstract}
This paper deals with perturbation theory for discrete spectra of linear operators. To simplify exposition we consider here self-adjoint operators. This theory is based on the Feshbach-Schur map and it has advantages with respect to the standard perturbation theory in three aspects: (a) it readily produces rigorous estimates on eigenvalues and eigenfunctions with explicit constants; (b) it is compact and elementary (it uses properties of norms and the fundamental theorem of algebra about solutions of polynomial equations); and (c) it is based on a self-contained formulation of a fixed point problem for the eigenvalues and eigenfunctions, allowing for easy iterations. We apply our abstract results to obtain rigorous bounds on the ground states of Helium-type ions.
\end{abstract}

Mathematics Subject Classification 2020. Primary 47A55, 35P15, 81Q15; Secondary 47A75, 35J10

Keywords. Perturbation theory, Spectrum, Feshbach-Schur map, Schroedinger operator, Atomic systems, Helium-type ions, Ground state

\section{Set-up and result}

The eigenvalue perturbation theory is a major tool in mathematical physics and applied mathematics. In the present form, it goes back to Rayleigh and Schrödinger and became

G. Dusson: Laboratoire de Mathématiques de Besançon, UMR CNRS 6623, Université Bourgogne Franche-Comté, 25030 Besançon, France; email: genevieve.dusson@math.cnrs.fr . Supported in part by the French "Investissements d'Avenir" program, project ISITE-BFC (contract ANR-15-IDEX-0003).

I.M. Sigal: Department of Mathematics, University of Toronto, Toronto, ON M5S 2E4, Canada; email: im.sigal@utoronto.ca. Supported in part by NSERC Grant No. NA7901.

B. Stamm: Center for Computational Engineering Science, RWTH Aachen University, 52062 Aachen, Germany; email: best@acom.rwth-aachen.de . 
a robust mathematical field in the works of Kato and Rellich. It was extended to quantum resonances by Simon, see [2, 5, 6, 11, 12, 13, 14, 15] for books and a book-size review.

A different approach to the eigenvalue perturbation problem going back to works of Feshbach and based on the Feshbach-Schur map was introduced in [1] and extended in $[4,3]$.

In this paper, we develop further this approach proposing a self-contained theory in a form of a fixed point problem for the eigenvalues and eigenfunctions. It is more compact and direct than the traditional one and, as we show elsewhere, extends to the nonlinear eigenvalue problem.

We show that this approach leads naturally to bounds on the eigenvalues and eigenfunctions with explicit constants, which we use in an estimation of the ground state energies of the Helium-type ions.

The approach can handle tougher perturbations, non-isolated eigenvalues (see [1, 4]) and continuous spectra as well as discrete ones. In this paper, we restrict ourselves to the latter. Namely, we address the eigenvalue perturbation problem for operators on a Hilbert space of the form

$$
H=H_{0}+W
$$

where $H_{0}$ is an operator with some isolated eigenvalues and $W$ is an operator, small relative to $H_{0}$ in an appropriate norm. The goal is to show that $H$ has eigenvalues near those of $H_{0}$ and estimate these eigenvalues.

Specifically, with $\|\cdot\|$ standing for the vector and operator norms in the underlying Hilbert space, we assume that

(A) $H_{0}$ is a self-adjoint, non-negative operator $\left(H_{0} \geq \beta>0\right)$;

(B) $W$ is symmetric and form-bounded with respect to $H_{0}$, in the sense that

$$
\left\|H_{0}^{-\frac{1}{2}} W H_{0}^{-\frac{1}{2}}\right\|<\infty
$$

(C) $H_{0}$ has an isolated eigenvalue $\lambda_{0}>0$ of a finite multiplicity, $m$.

Here $\|\cdot\|$ is the operator norm and $H_{0}^{-s}, 0<s<1$, is defined either by the spectral theory or by the explicit formula

$$
H_{0}^{-s}:=c \int_{0}^{\infty}\left(H_{0}+\omega\right)^{-1} \frac{d \omega}{\omega^{s}}
$$

where $c:=\left[\int_{0}^{\infty}(1+\omega)^{-1} \frac{d \omega}{\omega^{s}}\right]^{-1}$.

It turns out to be useful in the proofs below to use the following form-norm

$$
\|W\|_{H_{0}}:=\left\|H_{0}^{-\frac{1}{2}} W H_{0}^{-\frac{1}{2}}\right\|,
$$

Let $P$ be the orthogonal projection onto the span of the eigenfunctions of $H_{0}$ corresponding to the eigenvalue $\lambda_{0}$ and let $P^{\perp}:=\mathbf{1}-P$. Let $\gamma_{0}$ be the distance of $\lambda_{0}$ to 
the rest of the spectrum of $H_{0}$, and $\lambda_{*}:=\lambda_{0}+\gamma_{0}$. In what follows, we often deal with the expression

$$
\Phi(W):=\frac{\lambda_{0} \lambda_{*}}{\gamma_{0}}\left\|P^{\perp} W P\right\|_{H_{0}}^{2} .
$$

The following theorem proven in Section 2 is the main result of this paper.

Main Theorem 1.1. Let Assumptions $(A)-(C)$ be satisfied and assume that, for some $0<b<1$ and $0<a<1-b$,

$$
\begin{aligned}
\left\|P^{\perp} W P^{\perp}\right\|_{H_{0}} & \leq \frac{b \gamma_{0}}{\lambda_{*}}, \\
\|P W P\|+k \Phi(W) & <a \gamma_{0}, \\
k \Phi(W) & <\frac{1}{2}\left(a \gamma_{0}-\|P W P\|\right),
\end{aligned}
$$

where $k:=\frac{1}{1-a-b}$. Then the spectrum of the operator $H$ near $\lambda_{0}$ consists of isolated eigenvalues $\lambda_{i}$ of the total multiplicity $m$ satisfying, together with their normalized eigenfunctions $\varphi_{i}$, the following estimates

$$
\begin{aligned}
\left|\lambda_{i}-\lambda_{0}\right| & \leq\|P W P\|+k \Phi(W), \\
\left\|\varphi_{i}-\varphi_{0 i}\right\| & \leq k \sqrt{\frac{\Phi(W)}{\gamma_{0}}}
\end{aligned}
$$

where $\varphi_{0 i}$ are appropriate eigenfunctions of $H_{0}$ corresponding to the eigenvalue $\lambda_{0}$.

Remark 1 (Comparison with [3]). A similar result was already proven in [3]. Here, the theory is made self-contained and formulated as the fixed point problem and the bounds are tightened.

Remark 2 (Conditions on $W$ ) The fine tuning the conditions (1.3)-(1.5) on $W$ is used in application of this theorem to atomic systems in Section 3.

Note that because of the elementary estimate

$$
\Phi(W) \leq \frac{1}{\gamma_{0}}\left\|P W P^{\perp} W P\right\|
$$

see (3.11) below, the computation of $\|P W P\|$ and $\Phi(W)$ reduces to computing the largest eigenvalues of the simple $m \times m$-matrices $P W P,-P W P$ and $P W P^{\perp} W P$.

Remark 3 (Higher-order estimates and non-degenerate $\lambda_{0}$ ). In fact, one can estimate $\lambda_{i}$ (as well as the eigenfunctions $\varphi_{i}$ ) to an arbitrary order in $\left\|P^{\perp} W P\right\|_{H_{0}} / \gamma_{0}$. As a demonstration, we derive (after the proof of Theorem 1.1) the second-order estimate of the eigenvalue in the rank-one $(m=1)$ case

$$
\left|\lambda_{1}-\lambda_{0}-\langle W\rangle\right| \leq k \Phi(W)
$$


where $\varphi_{0}$ is the eigenfunction of $H_{0}$ corresponding to $\lambda_{0},\langle W\rangle:=\left\langle\varphi_{0}, W \varphi_{0}\right\rangle$.

For degenerate $\lambda_{0}$, we would like to prove a similar bound on $\left|\lambda_{i}-\lambda_{0}-\mu_{i}\right|$, where $\mu_{i}$ is the corresponding eigenvalue of the $m \times m$-matrix $P W P$. Here, we have a partial result (proven at the end of the next section) for the lowest eigenvalue $\lambda_{1}$ of $H$ :

$$
\lambda_{0}+\mu_{\min }-k \Phi(W) \leq \lambda_{1} \leq \lambda_{0}+\mu_{\min },
$$

where $\mu_{\min }$ denotes the smallest eigenvalue of the matrix $P W P$.

Remark 4 (Non-self-adjoint $H$ ). With the sacrifice of the explicit constants in (1.6) and (1.7) (mostly coming from (2.5)), the self-adjointness assumption on $H$ can be removed. However, for the problem of quantum resonances, one can still obtain explicit estimates.

In the rest of this section $H$ is an abstract operator not necessarily self-adjoint or of the form (1.1). Our approach is grounded in the following theorem (see [4], Theorem 11.1).

Theorem 1.2. Let $H$ be an operator on a Hilbert space and $P$ and $P^{\perp}$, a pair of projections such that $P+P^{\perp}=1$. Assume $H^{\perp}:=P^{\perp} H P^{\perp}$ is invertible on $\operatorname{Ran} P^{\perp}$ and the expression

$$
F_{P}(H):=P\left(H-H R^{\perp} H\right) P,
$$

where $R^{\perp}:=P^{\perp}\left(H^{\perp}\right)^{-1} P^{\perp}$, defines a bounded operator. Then $F_{P}$, considered as a map on the space of operators, is isospectral in the following sense:

(a) $\lambda \in \sigma(H)$ if and only if $0 \in \sigma\left(F_{P}(H-\lambda)\right)$;

(b) $H \psi=\lambda \psi$ if and only if $F_{P}(H-\lambda) \varphi=0$;

(c) $\operatorname{dim} \operatorname{Null}(H-\lambda)=\operatorname{dim} \operatorname{NullF}_{P}(H-\lambda)$.

(d) $\psi$ and $\varphi$ in (b) are related as $\varphi=P \psi$ and $\psi=Q_{P}(\lambda) \varphi$, where

$$
Q_{P}(\lambda):=P-P^{\perp}\left(H^{\perp}-\lambda\right)^{-1} P^{\perp} H P \text {. }
$$

Finally, $F_{P}(H)^{*}=F_{P^{*}}\left(H^{*}\right)$ and therefore, if $H$ and $P$ are self-adjoint, then so is $F_{P}(H)$.

A proof of this theorem is elementary and short; it can be found in [1], Section IV.1, pp 346-348, and [4], Appendix 11.6, pp 123-125.

The map $F_{P}$ on the space of operators, is called the Feshbach-Schur map. The relation $\psi=Q_{P}(\lambda) \varphi$ allows us to reconstruct the full eigenfunction from the projected one. By statement (a), we have 
Corollary 1.3. Assume there is an open set $\Lambda \subset \mathbb{C}$ such that $H-\lambda, \lambda \in \Lambda$, is in the domain of the map $F_{P}$, i.e. $F_{P}(H-\lambda)$ is well defined. Define the operator-family

$$
H(\lambda):=F_{P}(H-\lambda)+\lambda P,
$$

and let $v_{i}(\lambda)$, for $\lambda$ in $\Lambda$, denote its eigenvalues counted with multiplicities. Then the eigenvalues of $H$ in $\Lambda$ are in one-to-one correspondence with the solutions of the equations

$$
v_{i}(\lambda)=\lambda
$$

Concentrating on the eigenvalue problem, Corollary 1.3 shows that the original problem

$$
H \psi=\lambda \psi,
$$

is mapped into the equivalent eigenvalue problem,

$$
H(\lambda) \varphi=\lambda \varphi
$$

nonlinear in the spectral parameter $\lambda$, but on the smaller space Ran $P$.

Since the projection $P$ is of a finite rank, the original eigenvalue problem (1.13) is mapped into an equivalent lower-dimensional/finite-dimensional one, (1.14). Of course, we have to pay a price for this: at one step we have to solve a one-dimensional fixed point problem that can be equivalently seen as a non-linear eigenvalue problem and invert an operator in $\operatorname{Ran} P^{\perp}$.

We call this approach the Feshbach-Schur map method, or FSM method, for short. It is rather compact, as one easily see skimming through this paper and entirely elementary.

We call $H(\lambda)$ the effective Hamiltonian (matrix) and write as

$$
H(\lambda)=P H P+U(\lambda)
$$

with the self-adjoint effective interaction, or a Schur complement, $U(\lambda)$, defined as

$$
U(\lambda):=-P H P^{\perp}\left(H^{\perp}-\lambda\right)^{-1} P^{\perp} H P .
$$

It is shown in Lemma 2.1 below that (1.16) defines a bounded operator family.

We mention here some additional properties discussed in [3].

Proposition 1.4. Let $H$ be self-adjoint, let $\Lambda$ be the same as in Corollary 1.3, let $m$ be the rank of $P$ and let the eigenvalues of $H(\lambda), \lambda \in \Lambda \cap \mathbb{R}$, be labeled in the order of their increase counting their multiplicities so that

$$
v_{1}(\lambda) \leq \ldots \leq v_{m}(\lambda)
$$

Then we have that (a) a solution of the equation $v_{i}(\lambda)=\lambda$, for $\lambda \in \Lambda \cap \mathbb{R}$, is the $i$-th eigenvalue, $\lambda_{i}$, of $H$ and vice versa; (b) $v_{i}$ is differentiable in $\lambda$ and $v_{i}^{\prime}(\lambda) \leq 0$, for $\lambda \in \Lambda \cap \mathbb{R}$. 
Proof. (a) By (1.17), $\lambda_{i}=v_{i}\left(\lambda_{i}\right)<v_{i+1}(\lambda)=\lambda_{i+1}$ (except for the level crossings), which proves the result. For (b), by the explicit formula

$$
U^{\prime}(\lambda):=-P H P^{\perp}\left(H^{\perp}-\lambda\right)^{-2} P^{\perp} H P \leq 0,
$$

we have $U^{\prime}(\lambda) \leq 0$, which by the Hellmann-Feynman theorem (cf. (2.19)) implies $v_{i}^{\prime}(\lambda) \leq 0$.

Remark 5 (Perturbation expansion). In the context of Hamiltonians of form (1.1) satisfying Assumptions (A)-(C), the FSM leads to a perturbation expansion to an arbitrary order. Indeed, in this case, $P$ is the orthogonal projection onto $\operatorname{Null}\left(H_{0}-\lambda_{0}\right)$, $P^{\perp}:=\mathbf{1}-P$ and $U(\lambda)$ can be written as

$$
U(\lambda):=-P W P^{\perp}\left(H^{\perp}-\lambda\right)^{-1} P^{\perp} W P .
$$

Now, using the notation $A^{\perp}:=\left.P^{\perp} A P^{\perp}\right|_{\operatorname{Ran} P^{\perp}}$ and expanding

$$
\left(H^{\perp}-\lambda\right)^{-1}=\left(H_{0}^{\perp}+W^{\perp}-\lambda\right)^{-1}
$$

in $W^{\perp}$ and at the same time iterating fixed point equation (1.12), we generate a perturbation expansion for eigenvalues $H$ to an arbitrary order (see also Remark 2 above).

The paper is organized as follows. In Section 2, we present the proof of the main result, Theorem 1.1. Section 3 uses this result to obtain bounds of the ground-state energy of Helium-type ions.

\section{Perturbation estimates}

We want to use Theorem 1.2(b, c) to reduce the original eigenvalue problem to a simpler one. In this section, we assume that Conditions (A)-(C) of Section 1 are satisfied. Recall that $\gamma_{0}$ is the distance of $\lambda_{0}$ to the rest of the spectrum of $H_{0}$ and the expression $\Phi(W)$ is defined in (1.2). First, we prove that the operator $F_{P}(H-\lambda)$ is well-defined for $\lambda \in \Omega$, where, with $a$ the same as in Theorem 1.1,

$$
\Omega:=\left\{z \in \mathbb{C}:\left|z-\lambda_{0}\right| \leq a \gamma_{0}\right\} .
$$

Recall that $P$ denotes the orthogonal projection onto $\operatorname{Null}\left(H_{0}-\lambda_{0}\right)$ and $P^{\perp}:=\mathbf{1}-P$. Denote

$$
H^{\perp}:=\left.P^{\perp} H P^{\perp}\right|_{\operatorname{Ran} P^{\perp}} \quad \text { and } \quad R^{\perp}(\lambda):=P^{\perp}\left(H^{\perp}-\lambda\right)^{-1} P^{\perp}
$$

and recall $k=\frac{1}{1-a-b}$. We have

Lemma 2.1. Recall $\lambda_{*}:=\lambda_{0}+\gamma_{0}$ and assume (1.3). Then, for $\lambda \in \Omega$, the following statements hold

(a) The operator $H^{\perp}-\lambda$ is invertible on $\operatorname{Ran} P^{\perp}$; 
(b) The inverse $R^{\perp}(\lambda):=P^{\perp}\left(H^{\perp}-\lambda\right)^{-1} P^{\perp}$ defines a bounded, analytic operatorfamily;

(c) The expression

$$
U(\lambda):=-P H R^{\perp}(\lambda) H P
$$

defines a finite-rank, analytic operator-family, bounded as

$$
\|U(\lambda)\| \leq k \Phi(W) .
$$

(d) $U(\lambda)$ is symmetric for any $\lambda \in \Omega \cap \mathbb{R}$ and therefore $H(\lambda)$ is self-adjoint as well. Proof. With the notation $A^{\perp}:=\left.P^{\perp} A P^{\perp}\right|_{\operatorname{Ran} P^{\perp} \text {, we write }}$

$$
H^{\perp}=H_{0}^{\perp}+W^{\perp} \text {. }
$$

To prove (a), we let $H_{\lambda}:=H_{0}^{\perp}-\lambda$ and use the factorization $H_{\lambda}=\left|H_{\lambda}\right|^{\frac{1}{2}} V_{\lambda}\left|H_{\lambda}\right|^{\frac{1}{2}}$, where $V_{\lambda}$ is a unitary operator, and use that, for $\lambda \in \Omega$, the operator $H_{\lambda}$ is invertible and therefore we have the identity

$$
H^{\perp}-\lambda=\left|H_{\lambda}\right|^{\frac{1}{2}}\left[V_{\lambda}+K_{\lambda}\right]\left|H_{\lambda}\right|^{\frac{1}{2}},
$$

where $K_{\lambda}:=\left|H_{\lambda}\right|^{-\frac{1}{2}} W^{\perp}\left|H_{\lambda}\right|^{-\frac{1}{2}}$. Next, for $\lambda \in \Omega$, we have $\left\|H_{0}^{\perp}\left|H_{\lambda}\right|^{-1} P^{\perp}\right\|=f(\lambda)$, where

$$
f(\lambda):=\sup \left\{\left|\frac{s}{s-\lambda}\right|: s \geq 0,\left|s-\lambda_{0}\right| \geq \gamma_{0}\right\} .
$$

Assuming $\left|\lambda-\lambda_{0}\right| \leq a \gamma_{0}$ and using

$$
\left|\frac{s}{s-\lambda}\right| \leq 1+\left|\frac{\lambda}{s-\lambda}\right| \leq 1+\frac{\lambda_{0}+a \gamma_{0}}{(1-a) \gamma_{0}}=\frac{\lambda_{*}}{(1-a) \gamma_{0}},
$$

we obtain

$$
f(\lambda) \leq \frac{\lambda_{*}}{(1-a) \gamma_{0}}
$$

Since $H_{0}^{\frac{1}{2}}\left|H_{\lambda}\right|^{-\frac{1}{2}} P^{\perp}=\left(\left.H_{0}\left|H_{\lambda}\right|^{-1}\right|_{\operatorname{Ran} P^{\perp}}\right)^{\frac{1}{2}} P^{\perp}$, we have for $\lambda \in \Omega$,

$$
\left\|H_{0}^{\frac{1}{2}}\left|H_{\lambda}\right|^{-\frac{1}{2}} P^{\perp}\right\| \leq\left[\frac{\lambda_{*}}{(1-a) \gamma_{0}}\right]^{\frac{1}{2}}
$$

which implies in particular that $\left\|K_{\lambda}\right\| \leq \frac{\lambda_{*}}{(1-a) \gamma_{0}}\left\|W^{\perp}\right\|_{H_{0}}$. By the assumption (1.3), i.e., $\left\|W^{\perp}\right\|_{H_{0}} \leq \frac{b \gamma_{0}}{\lambda_{*}}$, we have

$$
\left\|K_{\lambda}\right\| \leq \frac{b}{1-a}
$$


Since $1-a>b$, by (2.4), the operator $H^{\perp}-\lambda$ is invertible and its inverse is analytic in $\lambda \in \Omega$, which proves (a) and (b).

We show that statement (c) is also satisfied. Since $P H_{0}=H_{0} P$ and $P P^{\perp}=0$, we have

$$
P H P^{\perp}=P W P^{\perp}, \quad P^{\perp} H P=P^{\perp} W P .
$$

These relations and definition (2.2) yield

$$
U(\lambda)=-P W R^{\perp}(\lambda) W P .
$$

Inverting (2.4) on $\operatorname{Ran} P^{\perp}$ and recalling the notation $R^{\perp}(\lambda):=P^{\perp}\left(H^{\perp}-\lambda\right)^{-1} P^{\perp}$ gives

$$
R^{\perp}(\lambda)=\left|H_{\lambda}\right|^{-\frac{1}{2}} P^{\perp}\left[V_{\lambda}+K_{\lambda}\right]^{-1} P^{\perp}\left|H_{\lambda}\right|^{-\frac{1}{2}}
$$

Now, using identity (2.8), estimate (2.5) and (2.6), we find, for $\lambda \in \Omega$,

$$
\left\|H_{0}^{\frac{1}{2}} R^{\perp}(\lambda) H_{0}^{\frac{1}{2}}\right\| \leq \frac{k \lambda_{*}}{\gamma_{0}} .
$$

Furthermore, by the eigen-equation $H_{0} P=\lambda_{0} P$, we have

$$
\left\|H_{0}^{\frac{1}{2}} P\right\|^{2}=\lambda_{0}
$$

Using expression (2.7) and estimates (2.9) and (2.10), we arrive at inequality (2.3). The analyticity follows from (2.7) and the analyticity of $R^{\perp}(\lambda)$.

For (d), since $H_{0}, W$ and $P$ are self-adjoint, then so are $U(\lambda)$, for any $\lambda \in \Omega \cap \mathbb{R}$, and, since $U(\lambda)$ is bounded, $H(\lambda)$ is self-adjoint as well.

Proof of Theorem 1.1. Let $\Omega$ be given by equation (2.1). Recall that, by Lemma 2.1 and Theorem 1.2, the $m \times m$ matrix-family $H(\lambda):=F_{P}(H-\lambda)+\lambda P$, with $F_{P}$ given in (1.10), is well defined, for each $\lambda \in \Omega$, and can be written as (1.15). Since $P H P=\lambda_{0} P+P W P$, Eq. (1.15) can be rewritten as

$$
H(\lambda)=\lambda_{0} P+P W P+U(\lambda)
$$

Eqs (2.3) and (2.11) imply the inequality

$$
\left\|H(\lambda)-\lambda_{0} P-P W P\right\| \leq k \Phi(W) .
$$

By a fact from Linear Algebra, for each $\lambda \in \Omega \cap \mathbb{R}$, the total multiplicity of the eigenvalues of the $m \times m$ self-adjoint matrix $H(\lambda)$ is $m$.

Denote by $v_{i}(\lambda), i=1,2, \ldots, m$, the eigenvalues of $H(\lambda)$, repeated according to their multiplicities. Eq (2.12) yields

$$
\left|v_{i}(\lambda)-\lambda_{0}\right| \leq\|P W P\|+k \Phi(W) \text {. }
$$


Indeed, let $P_{i}(\lambda)$ be the orthogonal projection onto $\operatorname{Null}\left(H(\lambda)-v_{i}(\lambda)\right)$. Then

$$
H(\lambda) P_{i}(\lambda)=v_{i}(\lambda) P_{i}(\lambda)
$$

which, due to (2.11) and $P_{i}(\lambda) P=P_{i}(\lambda)$, can be rewritten as

$$
\left(v_{i}(\lambda)-\lambda_{0}\right) P_{i}(\lambda)=(P W P+U(\lambda)) P_{i}(\lambda)
$$

Equating the operator norms of both sides of this equation and using (2.12) and $\left\|P_{i}(\lambda)\right\|=1$ gives $(2.13)$.

By Corollary 1.3, the eigenvalues of $H$ in the interval $\Omega \cap \mathbb{R}$ are in one-to-one correspondence with the solutions of the equations

$$
v_{i}(\lambda)=\lambda
$$

in $\Omega \cap \mathbb{R}$. If this equation has a solution, then, due to (2.13), this solution would satisfy (1.6). Thus, we address (2.14). Let

$$
\Omega^{\prime}:=\left\{z \in \mathbb{R}:\left|z-\lambda_{0}\right| \leq r \gamma_{0}\right\}
$$

with

$$
r:=\frac{1}{\gamma_{0}}(\|P W P\|+k \Phi(W)) .
$$

By our assumption (1.4), $r<a<1-b$.

Recall that by the definition, a branch point is a point at which the multiplicity of one of the eigenvalue families (branches) changes. One could think on a branch point as a point where two or more distinct eigenvalue branches intersect. Our next result shows that the eigenvalue branches of $H(\lambda)$ could be chosen in a differentiable way and estimates their derivatives.

Proposition 2.2. The following statements hold.

i) The eigenvalues $v_{i}(\lambda)$ of $H(\lambda)$ and the corresponding eigenfunctions can be chosen to be differentiable for $\lambda \in \Omega^{\prime}$.

ii) The derivatives $v_{i}^{\prime}(\lambda), \lambda \in \Omega^{\prime}$, are bounded as

$$
\left|v_{i}^{\prime}(\lambda)\right| \leq \frac{k}{a-r} \frac{\Phi(W)}{\gamma_{0}}
$$

iii) $v_{i}(\lambda)$ maps the interval $\Omega^{\prime}$ into itself.

Consequently, since by (1.5) the r.h.s. of (2.16) is $<1$, the equations $v_{i}(\lambda)=\lambda$ have unique solutions in $\Omega^{\prime}$. 
Proof. Proof of (i) for simple $\lambda_{0}$. For a simple eigenvalue $\lambda_{0}, P$ is a rank-one projection on the space spanned by the eigenvector $\varphi_{0}$ of $H_{0}$ corresponding to the eigenvalue $\lambda_{0}$ and therefore Eq. (2.11) implies that $H(\lambda)=v(\lambda) P$, with

$$
v(\lambda):=\lambda_{0}+\left\langle\varphi_{0},(W+U(\lambda)) \varphi_{0}\right\rangle .
$$

This and Lemma 2.1 show that the eigenvalue $v(\lambda)$ is analytic.

Proof of (i) for degenerate $\lambda_{0}$. We pick an arbitrary point $\mu$ in $\Omega^{\prime}$ and let $P_{\mu i}$ be the orthogonal projections onto the eigenspaces of $H(\mu)$ corresponding to the eigenvalues $v_{i}(\mu)$, i.e. for a fixed $i, P_{\mu i}$ projects on the spans of all eigenvectors with the same eigenvalue $v_{i}(\mu)$. We now show that, in a neighbourhood of $\mu$, the eigenfunctions $\chi_{i}(\lambda)$ can be chosen in a differentiable way. We introduce the system of equations for the eigenvalues $v_{i}(\lambda)$ and corresponding eigenfunctions $\chi_{i}(\lambda)$ :

$$
\begin{aligned}
v_{i}(\lambda) & =\frac{\left\langle\chi_{i}(\lambda), H(\lambda) \chi_{i}(\lambda)\right\rangle}{\left\|\chi_{i}(\lambda)\right\|^{2}}, \\
\chi_{i}(\lambda) & =\chi_{i}(\mu)-R^{\perp}\left(v_{i}(\lambda), \lambda\right) W_{\lambda} \chi_{i}(\mu),
\end{aligned}
$$

where

$$
R^{\perp}(v, \lambda):=P_{\mu i}^{\perp}\left(P_{\mu i}^{\perp} H(\lambda) P_{\mu i}^{\perp}-v\right)^{-1} P_{\mu i}^{\perp}, \quad W_{\lambda}:=U(\lambda)-U(\mu) .
$$

The expression on the right side of (2.18) is the $Q$-operator for $H(\lambda)$ and $P_{\mu i}$ defined according to (1.11) and applied to $\chi_{i}(\mu)$. Note that systems (2.17)-(2.18) for different indices $i$ are not coupled. Furthermore, since $\chi_{i}(\mu)$ and $R^{\perp}\left(v_{i}(\lambda), \lambda\right) W_{\lambda} \chi_{i}(\mu)$ are orthogonal, we have $\left\|\chi_{i}(\lambda)\right\| \geq\left\|\chi_{i}(\mu)\right\|$; and $\chi_{i}(\lambda)$ and $\chi_{j}(\lambda)$ are almost orthogonal for $i \neq j$. Assuming this system has a solution $\left(v_{i}(\lambda), \chi_{i}(\lambda)\right)$, we see that, by Theorem 1.2(d), with $P=P_{\mu i}, \chi_{i}(\lambda)$ are eigenfunctions of $H(\lambda)$ with the eigenvalues $v_{i}(\lambda)$ and (2.17) follows from the eigen-equation $H(\lambda) \chi_{i}(\lambda)=v_{i}(\lambda) \chi_{i}(\lambda)$.

For each $i$, we can reduce system (2.17)-(2.18) to the single equation for $v_{i}(\lambda)$, by treating $\chi_{i}(\lambda)$ in (2.17) as given by (2.18). This leads to the fixed point problem for the functions $v_{i}(\lambda)$ :

$$
v=F_{i}(v, \lambda),
$$

where

$$
\begin{aligned}
F_{i}(v, \lambda) & :=\frac{\left\langle\chi_{i}(v, \lambda), H(\lambda) \chi_{i}(v, \lambda)\right\rangle}{\left\|\chi_{i}(v, \lambda)\right\|^{2}}, \\
\chi_{i}(v, \lambda) & :=\chi_{i}(\mu)-R^{\perp}(v, \lambda) W_{\lambda} \chi_{i}(\mu) .
\end{aligned}
$$

Notice that since $P_{\mu i}^{\perp} H(\lambda) P_{\mu i}^{\perp}$ are self-adjoint, the resolvent $R^{\perp}(v, \lambda)$ and its derivatives in $v$ are uniformly bounded in a neighbourhood of $\left(v_{i}(\mu), \mu\right)$, which does not contain branch points, except, possibly, for $\mu$.

To be more specific, the resolvent $R^{\perp}(v, \lambda)$ is uniformly bounded in a neighbourhood $\Omega_{\mu i}$ of $\left(v_{i}(\mu), \mu\right)$ whether $\mu$ is a branch point or not, as long as $\Omega_{\mu i}$ does not contain any other branch point than $\mu$. 
Hence $F_{i}(v, \lambda)$ is differentiable in $v$ and $\lambda$ and $\left|\partial_{v} F_{i}(v, \lambda)\right| \rightarrow 0$, as $\lambda \rightarrow \mu$ (since $\partial_{v} \chi_{i}(v, \lambda)=R^{\perp}(v, \lambda)^{2} W_{\lambda} \chi_{i}(\mu)$ and therefore

$$
\left\|\partial_{v} \chi_{i}(v, \lambda)\right\| \leq\left\|R^{\perp}(v, \lambda)^{2}\left(H_{0}+\alpha\right)^{\frac{1}{2}}\right\|\left\|W_{\lambda}\right\|_{H_{0}, \alpha}\left\|\left(H_{0}+\alpha\right)^{\frac{1}{2}} \chi_{i}(\mu)\right\| \rightarrow 0,
$$

as $\lambda \rightarrow \mu)$. Moreover, $v_{i}(\mu)-F_{i}\left(v_{i}(\mu), \mu\right)=0$. Let $f_{i}(v, \lambda):=v-F_{i}(v, \lambda)$. Then, by the above, $f_{i}\left(v_{i}(\mu), \mu\right)=0$ and $\left|\partial_{v} f_{i}(v, \lambda)-1\right| \rightarrow 0$, as $\lambda \rightarrow \mu$. Thus, the implicit function theorem is applicable to the equation $f_{i}(v, \lambda)=0$ and shows that there is a unique solution $v_{i}(\lambda)$ in a neighbourhood of $\left(v_{i}(\mu), \mu\right)$ and that this solution is differentiable in $\lambda$.

Next, we have

Lemma 2.3. We have, for $\lambda \in \Omega^{\prime}$,

$$
v_{i}^{\prime}(\lambda)=\frac{\left\langle\chi_{i}(\lambda), U^{\prime}(\lambda) \chi_{i}(\lambda)\right\rangle}{\left\|\chi_{i}(\lambda)\right\|^{2}} .
$$

(Eq. (2.19) is closely related to the widely used Hellmann-Feynman theorem.)

Proof. Let $\hat{\chi}_{i}(\lambda):=\frac{\chi_{i}(\lambda)}{\left\|\chi_{i}(\lambda)\right\|}$. Writing equation (2.17) as

$$
v_{i}(\lambda)=\left\langle\hat{\chi}_{i}(\lambda), H(\lambda) \hat{\chi}_{i}(\lambda)\right\rangle
$$

and differentiating this with respect to $\lambda$, we obtain

$$
v_{i}^{\prime}(\lambda)=\left\langle\hat{\chi}_{i}(\lambda), H^{\prime}(\lambda) \hat{\chi}_{i}(\lambda)\right\rangle+\eta(\lambda)
$$

where $\eta(\lambda):=\left\langle\hat{\chi}_{i}^{\prime}(\lambda), H(\lambda) \hat{\chi}_{i}(\lambda)\right\rangle+\left\langle\hat{\chi}_{i}(\lambda), H(\lambda) \hat{\chi}_{i}^{\prime}(\lambda)\right\rangle$. Now, moving $H(\lambda)$ in the last term to the l.h.s. and using that $H(\lambda) \hat{\chi}_{i}(\lambda)=v_{i}(\lambda) \hat{\chi}_{i}(\lambda)$, we find

$$
\eta(\lambda)=v_{i}(\lambda)\left[\left\langle\hat{\chi}_{i}^{\prime}(\lambda), \hat{\chi}_{i}(\lambda)\right\rangle+\left\langle\hat{\chi}_{i}(\lambda), \hat{\chi}_{i}^{\prime}(\lambda)\right\rangle\right]=v_{i}(\lambda) \partial_{\lambda}\left\langle\hat{\chi}_{i}(\lambda), \hat{\chi}_{i}(\lambda)\right\rangle=0,
$$

which implies (2.19).

To prove Eq. (2.16), we use formula (2.19) above and the normalization of $\hat{\chi}_{i}(\lambda)$ to estimate $v_{i}^{\prime}(\lambda)$ as

$$
\left|v_{i}^{\prime}(\lambda)\right| \leq\left\|U^{\prime}(\lambda)\right\|
$$

To bound the r.h.s. of (2.20), we use the analyticity of $U(\lambda)$ in $\Omega$ and estimate (2.3). Indeed, by the Cauchy integral formula, we have

$$
\left\|U^{\prime}(\lambda)\right\| \leq \frac{1}{R \gamma_{0}} \sup _{\left|\lambda^{\prime}-\lambda\right|=R \gamma_{0}}\left\|U\left(\lambda^{\prime}\right)\right\|,
$$

with $R \leq a-r$, so that $\left\{\lambda^{\prime} \in \mathbb{C}:\left|\lambda^{\prime}-\lambda\right|<R \gamma_{0}\right\} \subset \Omega$, for $\lambda \in \Omega^{\prime}$. This, together with (2.3) and under the conditions of Lemma 2.1, gives the estimate

$$
\left\|U^{\prime}(\lambda)\right\| \leq \frac{k}{a-r} \frac{\Phi(W)}{\gamma_{0}},
$$


for $\lambda \in \Omega^{\prime}$. Combining Eqs. (2.20) and (2.21), we arrive at (2.16).

Due to the definition of $r$ after (2.15), we can rewrite estimate (2.13) as

$$
\left|v_{i}(\lambda)-\lambda_{0}\right| \leq r \gamma_{0}
$$

By (2.15), this shows that $v_{i}(\lambda)$ maps the interval $\Omega^{\prime}$ into itself which proves (iii).

Hence, under condition (1.4), the fixed point equations $\lambda=v_{i}(\lambda)$ have unique solutions on the interval $\Omega^{\prime}$, proving Proposition 2.2.

By Corollary 1.3, the eigenvalues of $H$ in $\Omega^{\prime}$ are in one-to-one correspondence with the solutions of the equations $v_{i}(\lambda)=\lambda$. By Proposition 2.2, these equations have unique solutions, say, $\lambda_{i}$. Then, estimate (2.13) implies inequality (1.6).

To obtain estimate (1.7), we recall from Theorem 1.2 that $Q\left(\lambda_{j}\right) \varphi_{0 j}=\varphi_{j}$, where the operator $Q(\lambda)$ is given by

$$
Q(\lambda):=\mathbf{1}-R^{\perp}(\lambda) P^{\perp} W P,
$$

$\lambda_{j}$ are the eigenvalues of $H$ and $\varphi_{0 j}$ are eigenfunctions of $H_{0}$ corresponding to $\lambda_{0}$. This gives

$$
\varphi_{0 j}-\varphi_{j}=\varphi_{0 j}-Q\left(\lambda_{j}\right) \varphi_{0 j}=R^{\perp}\left(\lambda_{j}\right) P^{\perp} W P
$$

Now, as in the derivation of (2.9), using identity (2.8), estimates (2.5) and

$$
\left\|\left|H_{\lambda}\right|^{-\frac{1}{2}}\right\| \leq \frac{1}{\sqrt{(1-r) \gamma_{0}}}
$$

and the estimate $\left\|K_{\lambda}\right\| \leq \frac{b}{1-a}$, see (2.6), we find, for $\lambda \in \Omega^{\prime}$,

$$
\left\|R^{\perp}(\lambda) H_{0}^{\frac{1}{2}}\right\| \leq \frac{k \lambda_{*}^{\frac{1}{2}}}{\gamma_{0}},
$$

noting that $r<a$. sing inequalities (2.23) and (2.10), we estimate the r.h.s. of (2.22) as

$$
\left\|R^{\perp}\left(\lambda_{j}\right) P^{\perp} W P\right\| \leq k \sqrt{\frac{\Phi(W)}{\gamma_{0}}} .
$$

This, together with (2.22), gives (1.7). This proves Theorem 1.1.

Remark 6. Differentiating (2.18) with respect to $\lambda$, setting $\lambda=\mu$, using $W_{\lambda=\mu}=0$ and $W_{\lambda}^{\prime}=U^{\prime}(\lambda)$ and changing the notation $\mu$ to $\lambda$, we find the following formula for $\chi_{i}^{\prime}(\lambda)$ :

$$
\chi_{i}^{\prime}(\lambda)=-R^{\perp}\left(v_{i}(\lambda), \lambda\right) U^{\prime}(\lambda) \chi_{i}(\lambda) .
$$

Finally we prove the relations (1.8) and (1.9) stated in the introduction. 
Proof of (1.8). Eq. (2.11) gives

$$
H(\lambda)=\left(\lambda_{0}+\langle W\rangle+\langle U(\lambda)\rangle\right) P,
$$

where we use the notation $\langle A\rangle:=\left\langle\varphi_{0}, A \varphi_{0}\right\rangle$. Since $P$ is a rank one projection, it follows that $H(\lambda)$ has only one eigenvalue and this eigenvalue is

$$
v_{1}(\lambda)=\lambda_{0}+\langle W\rangle+\langle U(\lambda)\rangle
$$

This formula and estimate (2.3) show that $v_{1}(\lambda)$ obeys the estimate

$$
\left|v_{1}(\lambda)-\lambda_{0}-\langle W\rangle\right| \leq k \Phi(W) .
$$

By Proposition 2.2, the eigenvalue $\lambda_{1}$ of $H$ satisfies the equation $\lambda_{1}=v_{1}\left(\lambda_{1}\right)$. Then the estimate above implies inequality (1.8).

Proof of $E q$ (1.9). Let $W$ be symmetric and denote by $\mu_{\min }$ the smallest eigenvalue of the matrix $P W P$. Then $U(\lambda) \leq 0$ and (2.11) implies $H(\lambda) \leq \lambda_{0} P+P W P$. This, together with inf $A \leq \inf B$ for $A \leq B$, gives the upper bound $v_{1}(\lambda) \leq \lambda_{0}+\mu_{\min }$ on the smallest eigenvalue-branch $v_{1}(\lambda)$ of $H(\lambda)$.

For the lower bound, Eqs (2.3) and (2.11) imply the following estimate

$$
\lambda_{0}+\mu_{\min }-k \Phi(W) \leq v_{1}(\lambda) .
$$

The last two estimates and the equation $v_{i}(\lambda)=\lambda($ see (1.12)) yield (1.9).

\section{Application: The ground state energy of the Helium- type ions}

In this section, we will use the inequalities obtained above to estimate the ground state energy of the Helium-type ions, which is the simplest not completely solvable atomic quantum system. For simplicity, we assume that the nucleus is infinitely heavy, but we allow for a general nuclear charge $z e, z \geq 1$. Then the corresponding Schrödinger operator (describing 2 electrons of mass $m$ and charge $-e$, and the nucleus of infinite mass and charge $z e$ ) is given by

$$
H_{z, 2}=\sum_{j=1}^{2}\left(-\frac{\hbar^{2}}{2 m} \Delta_{x_{j}}-\frac{\kappa z e^{2}}{\left|x_{j}\right|}\right)+\frac{\kappa e^{2}}{\left|x_{1}-x_{2}\right|},
$$

acting on the space $L_{s y m}^{2}\left(\mathbb{R}^{6}\right)$ of $L^{2}$-functions symmetric (or antisymmetric) w.r.t. the permutation of $x_{1}$ and $x_{2}{ }^{2}$. Here $\kappa=\frac{1}{4 \pi \varepsilon_{0}}$ is Coulomb's constant and $\varepsilon_{0}$ is the vacuum

\footnotetext{
${ }^{2}$ By the Pauli principle, the product of coordinate and spin wave functions should antisymmetric w.r.t. permutation of the particle coordinates and spins. Hence, in the two particle case, after separation of spin variables, coordinate wave functions could be either antisymmetric or symmetric.
} 
permittivity. For $z=2, H_{z, 2}$ describes the Helium atom, for $z=1$, the negative ion of the Hydrogen and for $2<z \leq 94$ (or $\leq 103$, depending on what one counts as stable elements), Helium-type positive ion. (We can call (3.1) with $z>103$ a $z$-ion.)

It is well known that $H_{z, 2}$ has eigenvalues below its continuum. Variational techniques give excellent upper bounds on the eigenvalues of $H_{z, 2}$, but good lower bounds are hard to come by. Thus, we formulate

Problem 3.1. Estimate the ground state energy of $H_{z, 2}$.

The most difficult case is of $z=1$, the negative ion of the hydrogen, and the problem simplifies as $z$ increases.

Here we present fairly precise bounds on the ground state energy of $H_{z, 2}$ implied by our actual estimates. However, the conditions under which these estimates are valid impose rather sever restrictions in $z$. We introduce the reference energy

$$
E^{\mathrm{ref}}:=\frac{\kappa^{2} e^{4} m}{\hbar^{2}}=\alpha^{2} m c^{2}
$$

(twice the ground state energy of the hydrogen, or 2Ry), where $c$ is the speed of light in vacuum and

$$
\alpha=\kappa \frac{e^{2}}{\hbar c}
$$

is the fine structure constant, whose numerical value, approximately $1 / 137$. Let $E_{\#}^{(z)}$ stand for either $E_{z, 2}^{\mathrm{sym}} / E^{\text {ref }}$ or $E_{z, 2}^{\mathrm{as}} / E^{\text {ref }}$, the ground state energy of $H_{z, 2}$ on either symmetric or anti-symmetric functions. We have

Proposition 3.2. Assume $z \geq 31.25$ for the symmetric space and $z \geq 170$ for the anti-symmetric one. Then the ground state energy of $H_{z, 2}$ is bounded as

$$
-c^{\#} z^{2}+w_{1}^{\#} z-\frac{10 w_{2}^{\#}}{\gamma_{0}^{\#}} \leq E_{\#}^{(z)} \leq-c^{\#} z^{2}+w_{1}^{\#} z,
$$

where, for symmetric functions, $c=1, \gamma_{0}=\frac{3}{8}$ and $w_{1} \approx 0.6$ and $w_{2} \approx 0.27$, defined in equations (3.13) and (3.15), and, for anti-symmetric functions, $c^{\text {as }}=\frac{5}{8}, \gamma_{0}^{\text {as }}=\frac{5}{72}$ and $w_{1}^{\text {as }} \approx 0.20$ and $w_{2}^{\text {as }} \approx 0.01$, defined in (3.12).

The approximate values of $w_{1}, w_{2}, w_{1}^{\text {as }}$ and $w_{2}^{\text {as }}$ are computed numerically in Appendix B. Here we report the stable digits of our computations.

The inequalities $z \geq 31.25$ and $z \geq 170$ come from condition (1.3), while estimates (3.2), from (1.8), with $b=0.8$ and $a=0.1$ (which give $k=10$ ).

Table 1 compares the result for symmetric functions with computations in quantum chemistry. (We did not find results for the antisymmetric space.)

We observe that, except for $z=50$, the results of $[16,17,18]$ lie in the interval provided by the estimation (3.2). 


\begin{tabular}{|l|ccccc|}
\hline$z$ & 10 & 20 & 30 & 40 & 50 \\
\hline \hline$-E_{\text {exact }}($ from $[16,17,18])$ & 93.9 & 387.7 & 881.4 & 1575.2 & 2468.9 \\
\hline main part $-E_{z, 2}^{\text {sym,lead }}$ in $(3.2)$ & 94 & 388 & 882 & 1576 & 2470 \\
\hline relative difference err $_{z}$ & $0.11 \%$ & $0.077 \%$ & $0.068 \%$ & $0.051 \%$ & $0.045 \%$ \\
\hline relative error term $\Delta_{z}$ in $(3.2)$ & $8.51 \%$ & $2.06 \%$ & $0.91 \%$ & $0.51 \%$ & $0.32 \%$ \\
\hline
\end{tabular}

Table 1: Comparison of non-rigorous computations (see $[8,9,16,17,18]$ ) with the main part $-E_{z, 2}^{\text {sym,lead }}:=z^{2}-w_{1} z$ in equation (3.2), its relative contribution of the error estimation $\Delta_{z}:=10 w_{2} /\left(-E_{z, 2}^{\text {sym,lead }} \gamma_{0}\right)$ and the relative difference defined by $\operatorname{err}_{z}:=\left|E_{\text {exact }}-E_{z, 2}^{\text {sym,lead }}\right| /\left(-E_{z, 2}^{\text {sym,lead }}\right)$.

For computations of the relativistic and QED contributions, see [10, 18, 19].

Now, we derive some consequences of estimates (3.2).

Let $z_{*}$ be the smallest $z$ for which the error bound in the symmetric case is less than or equal to the smallest explicit (subleading) term $w_{1}$. Inequality (3.2) shows that the latter bound is satisfied for $z$ such that $w_{1} z \geq \frac{10 w_{2}}{\gamma_{0}}$, which shows that $z_{*}=\frac{10 w_{2}}{w_{1} \gamma_{0}}$ and consequently,

$$
z_{*} \approx 12 \text {. }
$$

According to equation (3.2), the symmetric ground state energy is lower than the anti-symmetric one, if $-z^{2}+w_{1} z<-\frac{5}{8} z^{2}+w_{1}^{\text {as }} z-160 w_{2}^{\text {as }}$. Using the values $w_{1} \approx 0.6, w_{2} \approx 0.3, w_{1}^{\text {as }} \approx 0.20$ and $w_{2}^{\text {as }} \approx 0.01$, we find that, based on (3.2), the ground state is symmetric and therefore its spin is 0 for

$$
z \geq \frac{4}{3}\left(\frac{2}{5}+\sqrt{\frac{4}{25}+\frac{96}{5}}\right)=\frac{8}{3} \approx 2.6 .
$$

We conjecture that the symmetric ground state energy is lower than the anti-symmetric one for all $z$ 's.

Finally, note that on symmetric functions, the eigenvalue of the unperturbed operator is simple and on anti-symmetric ones, has the degeneracy 4 , see below.

Proof of Proposition 3.2. First, we rescale the Hamiltonian (3.1) as $x \rightarrow x / \mu$, with

to obtain

$$
\mu:=\frac{z \kappa e^{2} m}{\hbar^{2}}=\frac{z}{\text { the Bohr radius }},
$$

$$
U_{\mu}^{-1} H_{z, 2} U_{\mu}=\frac{z^{2} \kappa^{2} e^{4} m}{\hbar^{2}} H^{(z)},
$$

where $U_{\mu}: \psi(x) \rightarrow \mu^{3} \psi(\mu x)$ and $H^{(z)}$ is the rescaled Hamiltonian given by

$$
H^{(z)}=\sum_{j=1}^{2}\left(-\frac{1}{2} \Delta_{x_{j}}-\frac{1}{\left|x_{j}\right|}\right)+\frac{1 / z}{\left|x_{1}-x_{2}\right|} .
$$


Thus, it suffices to estimate the ground state energy of $H^{(z)}$. We consider

$$
W=\frac{1 / z}{\left|x_{1}-x_{2}\right|}
$$

as the perturbation and $\sum_{j=1}^{2}\left(-\frac{1}{2} \Delta_{x_{j}}-\frac{1}{\left|x_{i}\right|}\right)$ as the unperturbed operator.

First, we consider the rescaled Hamiltonian $H^{(z)}$ on the symmetric functions subspace. On symmetric functions, the ground state energy of $\sum_{j=1}^{2}\left(-\frac{1}{2} \Delta_{x_{j}}-\frac{1}{\left|x_{j}\right|}\right)$ is -1 (see below), so we shift the operator $H^{(z)}$ by $1+\beta$, for some $\beta>0$, so that

$$
H^{(z)}+1+\beta=H_{0}+W
$$

with

$$
H_{0}:=\sum_{j=1}^{2}\left(-\frac{1}{2} \Delta_{x_{j}}-\frac{1}{\left|x_{j}\right|}\right)+1+\beta \text { and } W=\frac{1 / z}{\left|x_{1}-x_{2}\right|} .
$$

Now, the ground state energy of $H_{0}$ is $\beta$ and we can use inequality (1.8) and Proposition 1.4(c) to estimate the ground state energy of $H^{(z)}+1+\beta$.

By the HVZ theorem, the spectrum of $H_{0}$ on symmetric functions consists of the continuum $\left[e_{1}+1+\beta, \infty\right)$ and the eigenvalues $e_{m}+e_{n}+1+\beta, m, n \geq 1$, where $e_{n}, n=$ $1,2, \ldots$, denote the discrete eigenvalues of the Hydrogen Hamiltonian $-\frac{1}{2} \Delta_{x}-\frac{1}{|x|}$. The eigenvalues $e_{n}, n=1,2, \ldots$, are known explicitly:

$$
e_{n}=-\frac{1}{2 n^{2}}
$$

with the multiplicities of $n^{2}$ and the corresponding eigenfunctions are given by $\mathrm{Eq}$ (A.1) below.

Then the ground state energy of $H_{0}$ is $\lambda_{0}=2 e_{1}+1+\beta=\beta$, as claimed, and the gap, $\gamma_{0}=e_{1}+e_{2}-2 e_{1}=e_{2}-e_{1}=3 / 8$.

Next, we show that the condition (1.3) is satisfied for $z \geq 31.25$. We begin with the really rough estimate:

$$
\frac{1}{\left|x_{1}-x_{2}\right|} \leq h_{x_{1}}+h_{x_{2}}+10
$$

where $h_{x}:=-\frac{1}{2} \Delta_{x}-\frac{1}{|x|}$. First, we use Hardy's inequality, $-\Delta \geq \frac{1}{4|x|^{2}}$, and the estimate $\frac{1}{4 m|x|^{2}}-\frac{1}{|x|} \geq-m$ to obtain

$$
-\frac{1}{m} \Delta-\frac{1}{|x|} \geq-m \text {. }
$$

Next, passing to the relative and centre-of-mass coordinates, $x-y$ and $\frac{1}{2}(x+y)$, we find

$$
-\Delta_{x}-\Delta_{y}=-2 \Delta_{x-y}-\frac{1}{2} \Delta_{\frac{1}{2}(x+y)} \geq-2 \Delta_{x-y}
$$


which, together with (3.5), yields

$$
\frac{1}{\left|x_{1}-x_{2}\right|} \leq-\frac{1}{2} \Delta_{x_{1}-x_{2}}+2 \leq-\frac{1}{4}\left(\Delta_{x_{1}}+\Delta_{x_{2}}\right)+2 \text {. }
$$

Now, we use $-\frac{1}{2} \Delta_{x}=h_{x}+\frac{1}{|x|} \leq h_{x}-\frac{1}{4} \Delta_{x}+4$ to obtain

$$
-\frac{1}{4} \Delta_{x} \leq h_{x}+4
$$

The last two inequalities yield (3.4). Eq. (3.4), together with the relation

$$
H_{0}=h_{x_{1}}+h_{x_{2}}+1+\beta
$$

implies the estimate:

$$
\frac{1}{\left|x_{1}-x_{2}\right|} \leq H_{0}+9-\beta \text {. }
$$

Now, we check the condition (1.3):

$$
\left\|P^{\perp} W P^{\perp}\right\|_{H_{0}} \leq \frac{b \gamma_{0}}{\lambda_{*}} .
$$

Using the last relation, we estimate

$$
\begin{aligned}
z\left(H_{0}^{\perp}\right)^{-\frac{1}{2}} W\left(H_{0}^{\perp}\right)^{-\frac{1}{2}} & \leq\left(H_{0}^{\perp}\right)^{-1}\left(H_{0}^{\perp}+9-\beta\right) \\
& =\mathbf{1}+(9-\beta)\left(H_{0}^{\perp}\right)^{-1} .
\end{aligned}
$$

Recalling that $\lambda_{0}=\beta$, we see that $H_{0}^{\perp} \geq \beta+\gamma_{0}$, so that the last inequality gives

$$
0 \leq\left(H_{0}^{\perp}\right)^{-\frac{1}{2}} W\left(H_{0}^{\perp}\right)^{-\frac{1}{2}} \leq \frac{1}{z} \frac{9+\gamma_{0}}{\beta+\gamma_{0}},
$$

provided $\beta<9$. This implies

$$
\left\|P^{\perp} W P^{\perp}\right\|_{H_{0}} \leq \frac{1}{z} \frac{9+\gamma_{0}}{\beta+\gamma_{0}} .
$$

Since $\lambda_{*}=\beta+\gamma_{0}$, condition (1.3) is satisfied, if $\frac{9+\gamma_{0}}{z} \leq b \gamma_{0}$, which gives $z \geq \frac{9+\gamma_{0}}{b \gamma_{0}}$. Since $\gamma_{0}=3 / 8$ and $b=0.8$, this implies $z \geq 31.25$. We will need the following lemma, whose proof is given after the proof of the proposition.

Lemma 3.3. Recall that $P$ is the orthogonal projection onto the eigenspace of $H_{0}$ corresponding to the lowest eigenvalue $\beta$. We have

$$
\langle W\rangle=\|P W P\|=\frac{w_{1}}{z} \quad \text { and } \quad \Phi(W) \leq \frac{w_{2}}{z^{2} \gamma_{0}},
$$

with, recall, $\langle W\rangle:=\left\langle\varphi_{0}, W \varphi_{0}\right\rangle$, where $\varphi_{0}$ is the eigenfunction of $H_{0}$ corresponding to $\lambda_{0}$. 
We check now the second condition of Theorem 1.1, (1.4). Recall the definition

$$
r:=\frac{\|P W P\|+k \Phi(W)}{\gamma_{0}} .
$$

Then condition (1.4) can be written as $r<a$. By Lemma 3.3,

$$
r \leq \frac{1}{\gamma_{0}}\left(\frac{w_{1}}{z}+\frac{k w_{2}}{z^{2} \gamma_{0}}\right)
$$

Recall the condition $z \geq 31.25$ and $k=10, w_{1} \approx 0.6$ and $w_{2} \approx 0.3$. Thus

$$
\frac{k w_{2}}{z^{2} \gamma_{0}} \leq \frac{w_{1}}{z} \frac{k w_{2}}{31.25 \gamma_{0} w_{1}} \leq 0.5 \frac{w_{1}}{z} \text {. }
$$

Then we find $r \leq 1.5 \frac{w_{1}}{z \gamma_{0}}$ and therefore condition (1.4) is satisfied if $a>1.5 \frac{w_{1}}{z \gamma_{0}}$, or (for $a=0.1$ )

$$
z>\frac{3}{2} \frac{8}{3} \frac{0.6}{a}=24
$$

This is less restrictive than $z \geq 31.25$.

For the third condition (1.5), recalling that $\lambda_{0}=\beta$, so that $\lambda_{*}=\gamma_{0}+\beta\left(=\lambda_{1}\right.$, the second eigenvalue of $H_{0}$ ) and using both relations in (3.8), we obtain that condition (1.5) is satisfied if $k w_{2} /\left(z^{2} \gamma_{0}\right)<\frac{1}{2}\left(a \gamma_{0}-w_{1} / z\right)$, which is equivalent to

$$
z>\frac{1}{2 a \gamma_{0}}\left(w_{1}+\sqrt{w_{1}^{2}+8 a k w_{2}}\right) .
$$

Using the values $\gamma_{0}=\frac{3}{8}, w_{1} \approx 0.6$ and $w_{2} \approx 0.3$, and $b=0.8$ and $a=0.1$, giving $k=10$, this shows that the latter inequality, and therefore (1.5), holds if $z>30$, which is less restrictive than $z \geq 31.25$. Thus, all three conditions are satisfied for $z \geq 31.25$.

Next, we use (1.8) to estimate the ground state energy, $E_{\mathrm{sym}}^{(z)}$ of $H^{(z)}$. The first and second relations in (3.8), together with (1.8) and the fact that $U(\lambda) \leq 0$, gives the following bounds

$$
-\frac{k w_{2}}{\gamma_{0} z^{2}} \leq E_{\mathrm{sym}}^{(z)}+1-\frac{w_{1}}{z} \leq 0 .
$$

which after rescaling gives (3.2).

Now, we consider the ground state of $H^{(z)}$ on anti-symmetric functions. In this case, the ground state energy of $\sum_{j=1}^{2}\left(-\frac{1}{2} \Delta_{x_{j}}-\frac{1}{\left|x_{i}\right|}\right)$ is $e_{1}+e_{2}=-\frac{5}{8}$ of multiplicity 4 , with the ground states

$$
\phi_{2 \ell k}\left(x_{1}, x_{2}\right):=\frac{1}{\sqrt{2}}\left(\psi_{100}\left(x_{1}\right) \psi_{2 \ell k}\left(x_{2}\right)-\psi_{2 \ell k}\left(x_{1}\right) \psi_{100}\left(x_{2}\right)\right),
$$

for $(\ell, k)=(0,0),(1,-1),(1,0),(1,1)$, where $\psi_{n \ell k}(x)$ for $\ell=0,1,2, \ldots, n-1, k=$ $-\ell,-\ell+1, \ldots, \ell, n=1,2, \ldots$, are the eigenfunctions of the Hydrogen-like Hamiltonian 
$-\frac{1}{2} \Delta_{x}-\frac{1}{|x|}$ corresponding to eigenvalues $e_{n}$, so that $\psi_{100}(x)=\psi_{1}(x)$, see Appendix A. Now, we define the unperturbed operator as

$$
H_{0}:=\sum_{j=1}^{2}\left(-\frac{1}{2} \Delta_{x_{j}}-\frac{1}{\left|x_{j}\right|}\right)+\frac{5}{8}+\beta,
$$

to have $\beta$ as the lowest eigenvalue of $H_{0}$.

For the gap, since the first two eigenvalues are

$$
e_{1}+e_{2}+\frac{5}{8}+\beta=\beta \quad \text { and } \quad e_{1}+e_{3}+\frac{5}{8}+\beta\left(<e_{2}+e_{2}+\frac{5}{8}+\beta\right),
$$

we have $\gamma_{0}^{\text {as }}=e_{1}+e_{3}-\left(e_{1}+e_{2}\right)=e_{3}-e_{2}$ giving $\gamma_{0}^{\text {as }}=\frac{5}{72}$.

For condition (1.3), note first that since now $H_{0}=h_{x_{1}}+h_{x_{2}}+\frac{5}{8}+\beta$, Eq. (3.4) implies

$$
\frac{1}{\left|x_{1}-x_{2}\right|} \leq H_{0}+9+\frac{3}{8}-\beta
$$

instead of (3.6), which shows that (3.7) should be modified as

$$
\left\|P^{\perp} W P^{\perp}\right\|_{H_{0}} \leq \frac{1}{z} \frac{9+\frac{3}{8}+\gamma_{0}^{\text {as }}}{\beta+\gamma_{0}^{\text {as }}},
$$

in the anti-symmetric case. Since $\lambda_{*}^{\text {as }}=\beta+\gamma_{0}^{\text {as }}$, condition (1.3) is satisfied, if $\frac{1}{z}\left(9+\frac{3}{8}+\right.$ $\left.\gamma_{0}^{\text {as }}\right) \leq b \gamma_{0}^{\text {as }}$, which gives

$$
z \geq \frac{9+\frac{3}{8}+\gamma_{0}^{\text {as }}}{b \gamma_{0}^{\text {as }}}
$$

Since $\gamma_{0}^{\text {as }}=\frac{5}{72}$ and $b=0.8$, this implies $z \geq 170$ for the anti-symmetric case.

We skip the verification of conditions (1.4) and (1.5), which are simpler and similar to that of the symmetric case.

To estimate the ground state energy, $E_{\mathrm{as}}^{(z)}$, of $H^{(z)}$ on anti-symmetric functions, we use bound (1.9). First, we claim that $\Phi(W)$ defined in (1.2) satisfies (cf. Remark 2)

$$
\Phi(W) \leq \frac{\mu_{\max }}{\gamma_{0}^{\text {as }}}
$$

where $\mu_{\max }$ is the largest eigenvalue of the positive, $4 \times 4$-matrix $P W P^{\perp} W P$. Indeed, recall that

$$
\Phi(W):=\frac{\lambda_{0} \lambda_{*}\left\|P^{\perp} W P\right\|_{H_{0}}^{2}}{\gamma_{0}^{\text {as }}}
$$

with $\lambda_{0}=\beta$ and observe that $\left\|P W P^{\perp}\right\|_{H_{0}}=\|A\|$, with $A:=H_{0}^{-\frac{1}{2}} P W P^{\perp} H_{0}^{-\frac{1}{2}}$. Using now the relations

$$
A A^{*}=H_{0}^{-\frac{1}{2}} P W P^{\perp} H_{0}^{-1} W P H_{0}^{-\frac{1}{2}},
$$


$H_{0}^{-\frac{1}{2}} P=\lambda_{0}^{-\frac{1}{2}} P\left(\right.$ since $\left.H_{0} P=\lambda_{0} P\right)$, and $H_{0}^{-1} P^{\perp} \leq \lambda_{*}^{-1} P^{\perp}$, we find

$$
A A^{*} \leq\left(\lambda_{0} \lambda_{*}\right)^{-1} P W P^{\perp} W P,
$$

which, together with $\|A\|=\left\|A^{*}\right\|=\left\|A A^{*}\right\|^{\frac{1}{2}}$, gives

$$
\left\|P W P^{\perp}\right\|_{H_{0}}^{2} \leq\left(\lambda_{0} \lambda_{*}\right)^{-1}\left\|P W P^{\perp} W P\right\| .
$$

Since $P W P^{\perp} W P \geq 0$, we have $\left\|P W P^{\perp} W P\right\|=\mu_{\max }$, which gives (3.10).

Hence, we have to compute the smallest eigenvalue $\mu_{\min }$ of the $4 \times 4$ matrix $P W P$ and the largest eigenvalue, $\mu_{\max }$, of the positive, $4 \times 4$-matrix $P W P^{\perp} W P$, see (1.9).

By scaling, it is easy to see that the matrices $P W P$ and $P W P^{\perp} W P$ are of the form $P W P=z^{-1} A$ and $P W P^{\perp} W P=z^{-2} B$, where $A$ and $B$ are $z$-independent $4 \times 4$ matrices. Hence

$$
\mu_{\min }=\frac{w_{1}^{\text {as }}}{z} \quad \text { and } \quad \mu_{\max }=\frac{w_{2}^{\text {as }}}{z^{2}},
$$

for some positive $z$-independent constants $w_{1}^{\text {as }}$ and $w_{2}^{\text {as }}$. These constants are computed in Appendix B. Now, we see that (1.9) and (3.10) give

$$
\frac{w_{1}^{\text {as }}}{z}-\frac{k w_{2}^{\text {as }}}{\gamma_{0}^{\text {as }} z^{2}} \leq E_{\mathrm{as}}^{(z)}+\frac{5}{8} \leq \frac{w_{1}^{\text {as }}}{z},
$$

which after rescaling and setting $k=10$ gives (3.2) in the antisymmetric case.

Proof of Lemma 3.3. The ground state energy, $e_{1}$, of $-\frac{1}{2} \Delta_{x}-\frac{1}{|x|}$ is non-degenerate, with the normalized ground state (known as the 1s wavefunction) given by (see e.g. [7], or Wiki, with $a_{0}$ replaced by 1 due to the rescaling above)

$$
\psi_{1}(x)=\frac{1}{\sqrt{\pi}} e^{-|x|} .
$$

Hence, the ground state energy, 0 , of $H_{0}$ is also non-degenerate, with the normalized ground state $\psi_{1}\left(x_{1}\right) \psi_{1}\left(x_{2}\right)$ (in the symmetric subspace).

First, we compute $\langle W\rangle$ and $\|P W P\|$. Let $(\psi \otimes \phi)(x, y):=\psi(x) \phi(y)$ and, for an operator $A$ on $L^{2}\left(\mathbb{R}^{6}\right)$, we denote $\langle A\rangle:=\left\langle\psi_{1} \otimes \psi_{1}, A \psi_{1} \otimes \psi_{1}\right\rangle$. In the symmetric case, $P W P=\langle W\rangle P$, where

$$
\langle W\rangle:=\left\langle\psi_{1} \otimes \psi_{1}, W \psi_{1} \otimes \psi_{1}\right\rangle=\frac{1}{z} \int_{\mathbb{R}^{6}} \frac{\left|\psi_{1}(x) \psi_{1}(y)\right|^{2}}{|x-y|} d x d y=: \frac{1}{z} w_{1} .
$$

Hence $\|P W P\|=\frac{w_{1}}{z}$. This gives the first relation in (3.8).

Now, using that $P^{\perp}=\mathbf{1}-P$, we compute

$$
P W P^{\perp} W P=P W^{2} P-P W P W P=\frac{w_{2}}{z^{2}} P,
$$


and

$$
\begin{aligned}
w_{2} & :=z^{2}\left(\left\langle W^{2}\right\rangle-\langle W\rangle^{2}\right) \\
& =\int_{\mathbb{R}^{6}} \frac{\left|\psi_{1}(x) \psi_{1}(y)\right|^{2}}{|x-y|^{2}} d x d y-\left(\int_{\mathbb{R}^{6}} \frac{\left|\psi_{1}(x) \psi_{1}(y)\right|^{2}}{|x-y|} d x d y\right)^{2} .
\end{aligned}
$$

By the Schwarz inequality and the normalization of $\psi_{1}$, we have $w_{2} \geq 0$, which, together with (3.14), gives

$$
\left\|P W P^{\perp} W P\right\|=\frac{w_{2}}{z^{2}} .
$$

Eqs. (3.11) and (3.16) imply the second relation in (3.8).

Acknowledgments. The second author is grateful to Volker Bach and Jürg Fröhlich for enjoyable collaboration. The authors are indebted to the anonymous referee for many useful suggestions and remarks.

\section{A The eigenfunctions of the Hydrogen-like Hamilto- nian}

The eigenfunctions $\psi_{n \ell k}(x)$ are given by (see Wiki, "Hydrogen atom" with $a_{0}$ replaced by 1 )

$$
\psi_{n \ell k}(x)=\sqrt{\left(\frac{2 z}{n}\right)^{3} \frac{(n-\ell-1) !}{2 n(n+\ell) !}} e^{-\rho / 2} \rho^{\ell} L_{n-\ell-1}^{2 \ell+1}(\rho) Y_{\ell}^{k}(\theta, \phi),
$$

for $\ell=0,1,2, \ldots, n-1, k=-\ell,-\ell+1, \ldots, \ell, n=1,2, \ldots$ Here $(r, \theta, \phi)$ are the polar coordinates of $x, \rho=\frac{2 z r}{n}, L_{n-\ell-1}^{2 \ell+1}(\rho)$ is a generalized Laguerre polynomial of degree $n-\ell-1$, and $Y_{\ell}^{k}(\theta, \phi)$ is a spherical harmonic function of the degree $\ell$ and order $k .^{3}$

\section{B The numerical approximation of the constants $w_{1}$, $w_{2}, w_{1}^{a s}$ and $w_{2}^{a s}$}

Let us first focus on $w_{1}$ and $w_{2}$. From the definition

$$
w_{1}:=\int_{\mathbb{R}^{6}} \frac{\left|\psi_{1}(x) \psi_{1}(y)\right|^{2}}{|x-y|} d y d x
$$

\footnotetext{
${ }^{3}$ Quoting from Wikipedia (https://en.wikipedia.org/wiki/Hydrogen_atom, 30.10.2020): “... the generalized Laguerre polynomials are defined differently by different authors. The usage here is consistent with the definitions used by [7], p. 1136, and Wolfram Mathematica. In other places, the Laguerre polynomial includes a factor of $(n+\ell)$ !. ... or the generalized Laguerre polynomial appearing in the hydrogen wave function is $L_{n+\ell}^{2 \ell+1}(\rho)$ instead."
} 


$$
w_{2}:=\int_{\mathbb{R}^{6}} \frac{\left|\psi_{1}(x) \psi_{1}(y)\right|^{2}}{|x-y|^{2}} d y d x-w_{1}^{2},
$$

it becomes clear that we need to compute terms of the form

$$
\begin{aligned}
C_{\alpha} & =\int_{\mathbb{R}^{6}} \frac{\left|\psi_{1}(x) \psi_{1}(y)\right|^{2}}{|x-y|^{\alpha}} d y d x \\
& =\int_{\mathbb{R}^{3}}\left|\psi_{1}(x)\right|^{2} \int_{\mathbb{R}^{3}} \frac{\left|\psi_{1}(x-z)\right|^{2}}{|z|^{\alpha}} d z d x,
\end{aligned}
$$

with $\alpha=1,2$. We thus introduce a numerical quadrature in order to approximate the values of these integrals. We do not aim within this work to obtain the most efficient implementation. We define a numerical quadrature grid in terms of spherical coordinates with integration points $x_{i, n}$ and weights $\omega_{i, n}$ given by

$$
x_{i, n}=r_{i} s_{n}, \quad \omega_{i, n}=h r_{i}^{3} \omega_{n}^{\text {leb }}
$$

with

$$
r_{i}=\exp \left(-\ln \left(R_{\max }\right)+(i-1) h\right), \quad i=1, \ldots, N_{r}, \quad h=\frac{2 \ln \left(R_{\max }\right)}{N_{r}-1}
$$

and where $\left\{s_{n}, \omega_{n}^{\text {leb }}\right\}$ denote Lebedev integration points on the unit sphere with $N_{\text {leb }}$ points. Thus, $N_{r}$ denotes the number of points along the $r$-coordinate, $R_{\max }$ the radius of the furthest points away from the origin.

We thus approximate $C_{\alpha}$ by

$$
C_{\alpha} \approx \sum_{i=1}^{N_{r}} \sum_{n=1}^{N_{\text {leb }}} \omega_{i, n}\left|\psi_{1}\left(x_{i, n}\right)\right|^{2} \Phi_{\alpha}\left(x_{i, n}\right)
$$

with

$$
\Phi_{\alpha}\left(x_{i, n}\right)=\sum_{j=1}^{N_{r}} \sum_{m=1}^{N_{\mathrm{leb}}} \omega_{j, m} \frac{\left|\psi_{1}\left(x_{i, n}-x_{j, m}\right)\right|^{2}}{\left|x_{j, m}\right|^{\alpha}} .
$$

Then, we approximate $w_{1} \approx C_{1}$ and $W_{2} \approx C_{2}-C_{1}^{2}$.

We now shed our attention to the constants $w_{1}^{\text {as }}$ and $w_{2}^{\text {as }}$ and we first introduce the bi-electronic integrals, for $(\ell, k),\left(\ell^{\prime}, k^{\prime}\right)=(0,0),(1,-1),(1,0),(1,1)$, by

$$
\begin{aligned}
& \mathrm{A}_{\ell, k}^{\ell^{\prime}, k^{\prime}}=\int_{\mathbb{R}^{3}} \psi_{2 \ell k}(x) \psi_{2 \ell^{\prime} k^{\prime}}(x) \int_{\mathbb{R}^{3}} \frac{\left|\psi_{1}(y)\right|^{2}}{|x-y|} d y d x, \\
& \mathrm{~B}_{\ell, k}^{\ell^{\prime}, k^{\prime}}=\int_{\mathbb{R}^{3}} \psi_{1}(x) \psi_{2 \ell^{\prime} k^{\prime}}(x) \int_{\mathbb{R}^{3}} \frac{\psi_{1}(y) \psi_{2 \ell k}(y)}{|x-y|} d y d x .
\end{aligned}
$$

Then, using the definition (3.9) and symmetry properties, we derive the following expressions for the matrix elements

$$
\mathrm{M}_{\ell, k}^{\ell^{\prime}, k^{\prime}}=z\left\langle\psi_{2 \ell k}|W| \psi_{2 \ell^{\prime} k^{\prime}}\right\rangle=\mathrm{A}_{\ell, k}^{\ell^{\prime}, k^{\prime}}-\mathrm{B}_{\ell, k}^{\ell^{\prime}, k^{\prime}} .
$$




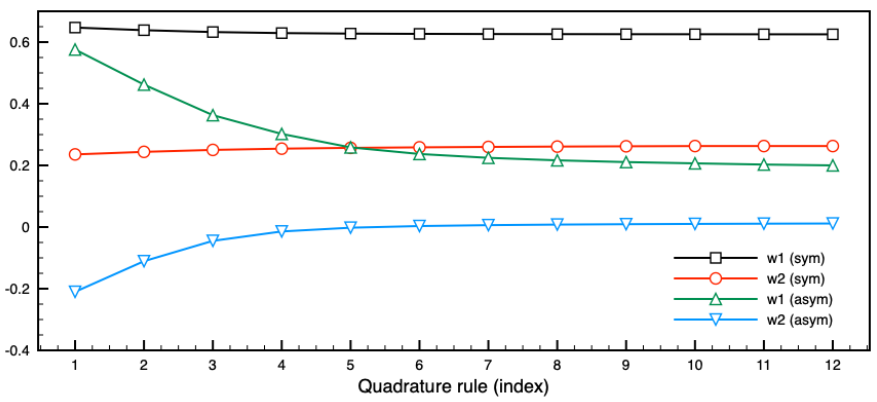

Figure 1: Value of the approximate coefficients $w_{1}, w_{2}, w_{1}^{\text {as }}$ and $w_{2}^{\text {as }}$ depending on the quadrature rule reported in Table 2.

We then employ the same quadrature rule as above to approximate the matrix elements $\mathrm{A}_{\ell, k}^{\ell^{\prime}, k^{\prime}}, \mathrm{B}_{\ell, k}^{\ell^{\prime}, k^{\prime}}$ and compute the smallest eigenvalue of $\mathrm{M}$ as an approximation of $w_{1}^{\text {as }}$.

Finally, the approximation of $w_{2}^{\text {as }}$ involves the matrix

$$
P W P^{\perp} W P=P W^{2} P-P W P W P,
$$

whose elements are given by $\mathrm{Q}=\mathrm{N}-\mathrm{M}^{2}$ with

$$
\mathrm{N}_{\ell, k}^{\ell^{\prime}, k^{\prime}}=z^{2}\left\langle\psi_{2 \ell k}\left|W^{2}\right| \psi_{2 \ell^{\prime} k^{\prime}}\right\rangle=\mathrm{C}_{\ell, k}^{\ell^{\prime}, k^{\prime}}-\mathrm{D}_{\ell, k}^{\ell^{\prime}, k^{\prime}}
$$

and

$$
\begin{aligned}
& \mathrm{C}_{\ell, k}^{\ell^{\prime}, k^{\prime}}=\int_{\mathbb{R}^{3}} \psi_{2 \ell k}(x) \psi_{2 \ell^{\prime} k^{\prime}}(x) \int_{\mathbb{R}^{3}} \frac{\left|\psi_{1}(y)\right|^{2}}{|x-y|^{2}} d y d x, \\
& \mathrm{D}_{\ell, k}^{\ell^{\prime}, k^{\prime}}=\int_{\mathbb{R}^{3}} \psi_{1}(x) \psi_{2 \ell^{\prime} k^{\prime}}(x) \int_{\mathbb{R}^{3}} \frac{\psi_{1}(y) \psi_{2 \ell k}(y)}{|x-y|^{2}} d y d x .
\end{aligned}
$$

We then again, approximate the different integrals with the above introduced quadrature rule and find the maximal eigenvalue of $Q$ in order to approximate $w_{2}^{\text {as }}$.

In Figure 1, we plot the the approximate value of $w_{1}, w_{2}, w_{1}^{\text {as }}$ and $w_{2}^{\text {as }}$ for different values of the parameters $R_{\max }, N_{r}$ and $N_{\text {leb }}$ given in Table 2 .

We obtain approximate values

$$
w_{1} \approx 0.625, \quad w_{2} \approx 0.2628, \quad w_{1}^{\mathrm{as}} \approx 0.3, \quad w_{2}^{\mathrm{as}} \approx 0.01
$$

where the last reported digit is stable over the last three quadrature rules. 


\begin{tabular}{|c|cccccccccccc|}
\hline index & 1 & 2 & 3 & 4 & 5 & 6 & 7 & 8 & 9 & 10 & 11 & 12 \\
\hline \hline$R_{\max }$ & 16 & 18 & 20 & 22 & 24 & 26 & 28 & 30 & 32 & 34 & 34 & 34 \\
\hline$N_{r}$ & 10 & 12 & 14 & 16 & 18 & 20 & 22 & 24 & 26 & 28 & 30 & 32 \\
\hline$N_{\text {leb }}$ & 194 & 266 & 350 & 590 & 974 & 1454 & 2030 & 2702 & 3470 & 4334 & 5294 & 5810 \\
\hline
\end{tabular}

Table 2: Parameters for the different quadrature rules.

\section{References}

[1] V. Bach, J. Fröhlich and I.M. Sigal, Quantum electrodynamics of confined non-relativistic particles. Adv. Math., 137(2):299-395, 1998.

[2] H. Cycon, R. Froese, W. Kirsch, B. Simon, Schrödinger Operators (with Applications to Quantum Mechanics and Global Geometry. Springer, 1987.

[3] G. Dusson, I.M. Sigal and B. Stamm, Analysis of the Feshbach-Schur method for the planewave discretizations of Schrödinger operators. arXiv:2008.10871.

[4] S. Gustafson and I.M. Sigal, Mathematical Concepts of Quantum Mechanics. Springer Science \& Business Media, Sept. 2011.

[5] P. Hislop and I.M. Sigal, Introduction to Spectral Theory. With Applications to Schrödinger Operators. Springer-Verlag, 1996.

[6] T. Kato, Perturbation theory for linear operators. Springer-Verlag, 1976.

[7] A. Messiah, Quantum Mechanics. New York: Dover. (1999).

[8] H. Nakashima, H. Nakatsuji, Solving the Schrödinger equation for helium atom and its isoelectronic ions with the free iterative complement interaction (ICI) method, J. Chem. Phys. 127, 224104 (2007).

[9] H. Nakashima, H. Nakatsuji, Solving the electron-nuclear Schrödinger equation of helium atom and its isoelectronic ions with the free iterativecomplement-interaction method. J. Chem. Phys. 128, 154107 (2008).

[10] K. Pachucki, V. Patkos and V.A. Yerokhin, Testing fundamental interactions on the helium atom. Phys. Rev. A95 (2017) 062510.

[11] M. Reed and B. Simon, Methods of Modern Mathematical Physics, Vol. II. Fourier Analysis and Self-Adjointness. Academic Press, 1975.

[12] M. Reed and B. Simon, Methods of Modern Mathematical Physics IV: Analysis of Operators. Academic Press, 1979.

[13] F. Rellich, Perturbation Theory of Eigenvalue Problems. Gordon and Breach: New York, 1969. 
[14] B. Simon, A Comprehensive Course in Analysis, Part 4: Operator Theory (American Mathematical Society, Providence, RI, 2015).

[15] B. Simon, Tosio Kato's work on non-relativistic quantum mechanics: Part 1 and 2, Bulletin of Mathematical Sciences, Vol 8 (2018), 121-232 and Vol 9 (2019) 1950005 (105 pages).

[16] A. V. Turbiner and J. C. L. Vieyra, Helium-like and Lithium-like ions: Ground state energy. arXiv:1707.07547v3, 2017.

[17] A. V. Turbiner, J. C. L. Vieyra, J.C. del Valle, and DJ Nader, Ultra-compact accurate wave functions for He-like and Li-like iso?electronic sequences and variational calculus: I. Ground state. International Journal of Quantum Chemistry, e26586, 2020; A. V. Turbiner, J. C. L. Vieyra and J.C. del Valle, Ultracompact accurate wave functions for He-like and Li-like iso-electronic sequences and variational calculus. I. Ground state. arXiv:2007.11745v, 2020.

[18] A.V. Turbiner, J.C. L. Vieyra, H. Olivares-Pilón, Few-electron atomic ions in non-relativistic QED: The ground state. Annals of Physics 409 (2019) 167908

[19] V.A. Yerokhin and K. Pachucki, Theoretical energies of low-lying states of light helium-like ions. Phys Rev A81 (2010) 022507. 